\title{
Tangence
}

\section{La rupture de la quotidienneté}

\section{Sylvie Dion}

Numéro 37, septembre 1992

Autopsie du fait divers

URI : https://id.erudit.org/iderudit/025721ar

DOI : https://doi.org/10.7202/025721ar

Aller au sommaire du numéro

Éditeur(s)

Tangence

ISSN

1189-4563 (imprimé)

1710-0305 (numérique)

Découvrir la revue

Citer cet article

Dion, S. (1992). La rupture de la quotidienneté. Tangence, (37), 8-15.

https://doi.org/10.7202/025721ar d'utilisation que vous pouvez consulter en ligne.

https://apropos.erudit.org/fr/usagers/politique-dutilisation/ 


\section{Du fait divers \\ et de ses usages}




\section{La rupture de la quotidienneté}

Sylvie Dion *

En face de l'événement banal, de la quotidienneté, des actualités politiques et économiques, les faits divers nous ouvrent la porte de l'étrangeté, du macabre et du mystère. Ils nous présentent un point de vue autre sur le monde, une vision du monde souvent irrationnelle, à la limite parfois de l'intelligible, porteuse des craintes et des convictions de l'époque dans laquelle ils s'inscrivent. Les faits divers sont des pratiques discursives qui font partie de l'univers de l'information journalistique et des mass media. Ils racontent l'histoire de gens ordinaires qui ont vécu des événements extraordinaires. Toutefois, quel que soit leur degrế d'aberration, ils demeurent étroitement liés à la réalité et au vécu. Mystère et réalité, c'est dans l'union de ces deux termes que les faits divers puisent leur pouvoir de fascination.

\section{" Le sang à la une ": de la chronique à l'hebdomadaire spécialisé}

Le terme :fait divers" signifie à la fois l'événement qui s'est produit et le texte qui en rend compte; il désigne la rubrique d'un quotidien sous laquelle on regroupe les incidents du jour, las meurtres, les accidents, les suicides ou tout autre événement marquant de la journée. L'emploi du terme *fait divers * remonte fin du XIXe siècle au moment de la création de la presse de masse en France. Dès la naissance de la presse populaire à un sou se produisit un changement notable dans les habitudes de consommation et de diffusion des faits divers. Remplaçant le traditionnel vendeur de canards et de complaintes, le journal sort

de son isolement aristocratique et, en abaissant son prix de vente pour se mettre à la portée de toutes les bourses, cherche à conquérir l'immense public populaire qu'il n'avait pu atteindre jusque-là. ${ }^{1}$

- Université du Québec à Rimouski et CELAT.

1 Musée national des arts et traditions populaires (France), Le fait divers [rédigé par Alain Monestier et Jacques Cheyronnaud], Paris, Editions de la Réunion des Musées nationaux, 1982, 166 p. (Exposition tenue à 
En plus d'être abordables pour les petites gens, ce qui n'était pas le cas des "gazettes * et des "nouvelles à main *, ces journaux offraient au grand public *une information essentiellement composée de faits divers présentés d'une manière romancée et volontiers mélodramatique ${ }^{2}$. Au Québec, il faudra attendre la publication de l'hebdomadaire Le petit journal en 1926 et celle du quotidien L'illustration en 1930 avant de parler de presse populaire. En effet, la presse canadienne-française à ses débuts et pendant longtemps sera la porte-parole de l'élite intellectuelle, du clergé et des nationalistes. Elle ne s'intéressera pas à l'homme ordinaire et, réciproquement, ce dernier consommera peu de journaux.

Outre les faits divers publiés dans les grands quotidiens comme La presse, Le soleil ou France soir, il existe des journaux spécialisés dans le fait divers appelés également *hebdomadaires d'actualité policière. . Ce sont ni plus ni moins des recueils de faits divers illustrés. Formule vieille de plus d'un siècle et mise sur pied d'abord par les canardiers français en réponse à la trop grande popularité des gazettes populaires,

cette littérature de colportage ayant été bien accueillie, les industriels de la presse en recopièrent à leur tour la formule. Ils inventèrent les hebdomadaires de faits divers illustrés, en adaptant à l'actualité les vieux procédés des canardiers ${ }^{3}$.

$\mathrm{Au}$ Québec, il existe actuellement plusieurs de ces publications. Les plus connues sont Allo police, Hebdo police et Pboto police. En France, la plus célèbre est sans doute Détective, créée en 1928 et qui avait à ses débuts Joseph Kessel comme directeur-rédacteur en chef. Les hebdomadaires Allo police et Hebdo police sont tirés à 75000 exemplaires environ. Allo police fut le premier journal québécois de ce genre. Il a vu le jour le 28 février 1953. Abondamment illustré et volontiers moraliste ${ }^{4}$, cet hebdomadaire se définissait comme un journal d'information sur le crime et de valorisation du travail des policiers. Dans sa première édition, le journal se présente ainsi:

Paris, au Musée des arts et traditions populaires, du 19 novembre 1982 au 18 avril 1983; catalogue de l'exposition, p. 114).

2 Ibid.

3 Jean-Claude Baillon, :Complaintes et canards ", Faits divers, Autrement, $\mathrm{n}^{\circ} 98$, avril 1988, p. 13.

4 À titre d'exemple, voir Allo Police du 8 septembre 1963, p. 2. Le journal s'y définit ainsi: " La ligne de conduite de notre journal est de mettre davantage en garde les jeunes comme les adultes contre les risques que comporte l'abandon d'une conduite saine et normale." 
10

Nous offrons aujourd'hui au public un nouveau journal, qui lui permettra de s'intéresser plus complètement à la magnifique tâche qu'accomplissent nos corps policiers pour protéger les citoyens. Cette publication est en quelque sorte une protestation contre les feuilles, souvent "importées", qui se complaisent dans l'exploitation sans vergogne des plus bas instincts de l'humanité. Allo Police ne se donne pas pour mission d'exposer les dessous des crimes à seule fin d'y trouver un sujet d'excitation [...]. ${ }^{5}$

À ses débuts, Allo police publiait conjointement les crimes de la semaine et les causes célèbres québécoises, américaines ou européennes sous forme de rubrique. Petit à petit, le journal se concentra sur l'actualité avec un goût de plus en plus prononcé pour les photographies choc et les délits à caractère sexuel tout en privilégiant les faits divers locaux. D'autres journaux, comme Hebdo police, plus près de la tradition des premiers recueils de faits divers, alignent les gros titres en page couverture, mêlent indifféremment actualités policières locales et faits divers venus de l'étranger, nomment rarement leurs sources et situent les récits de manière vague et imprécise ${ }^{6}$. L'avènement récent, au Québec, d'émissions télévisées consacrées aux faits divers $(911,24 / 24)$ calquées sur les modèles américains n'a fait qu'accentuer la tendance de ces journaux à mettre au premier plan des événements à caractère sexuel. Le fait divers n'est plus seulement accompagné de photographies, il l'est désormais de reportages télévisés.

\section{Notre fait divers quotidien}

Les versions d'un fait divers que l'on retrouve dans les quotidiens doivent être distinguées de celles, beaucoup plus longues et détaillées, qu'en offrent les journaux spécialisés. Nous ne nous occuperons ici que des premières. Certaines d'entre elles occupent les premières pages du journal (le plus souvent, les crimes locaux)

5 Allo Police, vol. I, $\mathrm{n}^{\circ} 1,1953$.

6 Hebdo police du 14 au 21 janvier 1989 annonce en page de titre quatre drames qui ne comportent aucune indication précise de lieux ou de temps: "Pour de l'argent, une infirmière empoisonne des patients "; "Sa fille est défigurée par un chien il tue la bête et le maître "; À 14 ans, violée et poignardée "; *Victime de la vengeance des homosexuels .. Ces gros titres renvoient à des articles qui racontent l'événement en détail. Chaque récit est appuyé de photographies. Toutefois, la situation géographique et temporelle demeure vague. De même, les protagonistes sont simplement identifiés par des prénoms. 
et d'autres se retrouvent au centre du journal et parfois même parmi les pages sportives (en général, les faits divers étrangers). Les faits divers «locaux * sont ceux qui concernent directement une collectivité donnée (le protagoniste appartient à la collectivité, l'événement se produit dans l'environnement immédiat du lecteur). De ce fait, de nombreux éléments d'information du texte ne pourront être correctement interprétés que par les destinateurs spécifiques auxquels le récit s'adresse, que par les initiés (lieux connus, pratiques courantes, etc.). Évidemment, le sens littéral du récit demeure intelligible en dehors de la collectivité. Toutefois, le fait divers "local. est aussi et surtout porteur des éléments d'informations de nature pragmatique qui échappent au destinateur non concerné. Le récit fait appel implicitement et explicitement à la compétence culturelle du lecteur. Les récits d'événements qui se passent à l'étranger concernent la collectivité dans la mesure où ils confortent nos préjugés sur l'Autre et attirent par leur exotisme et leur bizarrerie. Le fait divers d'origine étrangère crée, chez le lecteur autochtone, une distance vis-à-vis de l'événement. Loin de n'être qu'amusants ou distrayants, ces récits qui viennent d'ailleurs contribuent à façonner l'idée qu'une collectivité se fait des autres collectivités.

\section{Les visages de la transgression}

Le fait divers a mauvaise réputation. On parle souvent de manière méprisante de * chronique des chiens écrasés . Cette pratique discursive est le prototype du discours de violence. Une première caractéristique du récit de faits divers est de se présenter sous les traits d'une histoire véridique et familière. Associés à l'horreur, les récits de fait divers ont fréquemment pour thèmes les morts violentes, les meurtres odieux, les suicides. On retrouve également tous les types d'accidents, du plus cocasse au plus affreux, et aussi tous les types d'infractions à la loi, de la petite escroquerie à la découverte de vermine dans un restaurant. Les récits de faits divers rapportent aussi les catastrophes naturelles, présentent des monstres, des personnages anormaux, des manifestations de l'au-delà. À l'occasion, on y retrouve des actes héroïques et des erreurs judiciaires.

Certains thèmes pourront s'inscrire dans une série: drames familiaux où le père assassine femme et enfants pour ensuite s'enlever la vie, enfants mordus par des chiens de combats, viols concentrés dans une région, empoisonnement dans des restaurants 
12

asiatiques. Cette inscription en série laissera une impression de contagion, d'épidémie criminelle; en se multipliant ainsi, le crime inquiète davantage. Il devient incontrôlable et menaçant, s'inscrivant dans une sorte de malédiction.

La lecture d'un fait divers laisse une impression de proximité et d'actualité. Cette impression est liée à l'événement vécu. Contrairement à tout autre récit fictif, il est établi dès le départ que ce qui est raconté est non seulement véridique mais aussi réel. L'aspect dramatique, spectaculaire et mystérieux de ces récits étonne. Ils ne se limitent pas à l'énoncé pur et simple. La mise en récit organise et structure l'événement. L'énonciateur fait des choix en privilégiant une information plutôt qu'une autre. Il laisse des questions sans réponse et des affirmations en suspens; l'énonciateur sème le doute dans l'esprit du lecteur. Ce type de discours construit et diffuse des valeurs normatives, des croyances populaires et les préjugés qui hantent l'esprit du destinateur qu'il vise.

Le récit de fait divers est celui d'une transgression, d'un écart par rapport à une norme (sociale, morale, religieuse, naturelle). La transgression est le résultat d'une action qui consiste à désobéir, à violer un interdit, à dépasser les limites habituellement permises et tolérées. Cette transgression a mille visages. Elle va de la transgression sociale, la violation des lois civiles, morales, religieuses, à la transgression au sens plus large, c'est-à-dire la déviance sous toutes ses formes: déviance de la nature (tremblement de terre, inondation, monstre marin, monstre humain, etc.), déviance par intervention du surnaturel (apparition d'un saint ou de la Vierge, possession diabolique, maison hantée etc.), déviance morale et sociale (meurtre, inceste, etc.). Par là même, le fait divers est discours d'exclusion, de marginalisation. Ces récits nomment les préoccupations sociales, les tensions et les interdits tout en réaffirmant le code et les lois tant sociales que morales. La déviance peut être négative ou positive, morale ou sociale ou les deux à la fois. Elle peut être religieuse, relever de la superstition ou d'une croyance plus profane. Récits didactiques, les faits divers enseignent les bons et les mauvais comportements. Ils enseignent également la sanction et le châtiment qui résultent de la transgression. Des sanctions positives et des sanctions négatives accompagnent des transgressions positives ou négatives. Les transgressions et les sanctions négatives sont celles qui sont le plus souvent rapportées.

Le fait divers s'alimente à la marginalité quand il ne la nourrit pas. Il fait partie d'un processus d'apprentissage des valeurs nor- 
matives en procurant aux lecteurs une sorte de compétence événementielle, c'est-à-dire une compétence basée sur le récit d'un événement vrai ou présenté comme tel. Il donne "l'occasion de jouer au "comme si" "7. Dans l'esprit de l'énonciateur aussi bien que dans l'esprit de l'énonciataire, le fait que l'histoire racontée soit à la fois banale et véridique permet à ce dernier de récupérer le récit et de le vivre par personne interposée. Pour le sociologue Georges Auclair, l'attirance, le pouvoir de fascination des faits divers, ce qu'on qualifie souvent de curiosité "morbide",

ferait [...] appel aux sentiments les plus archaïques de l'homme: par la vue, le toucher, la possession d'un objet ou quelquefois la seule lecture, s'assimiler un peu de l'aura du tragique ou du sanglant, ce serait virtuellement en inverser le signe, de maléfique le rendre possiblement bénéfique ${ }^{8}$.

Cette illusion de proximité que renvoie le fait divers est révélatrice des rapports entre événement et réalité, d'une part, et récit et réception, d'autre part. En effet, une tragédie, un accident dramatique, un meurtre, etc., sont des faits divers dans la mesure où ils arrivent à quelqu'un d'autre que nous ou à quelqu'un d'autre qu'un membre de notre communauté. En d'autres termes, notre tragédie est souvent le fait divers d'un autre et vice versa. Tout en se voulant près du lecteur, le fait divers peut créer en même temps un sorte de distance. Prenons l'exemple des événements tragiques survenus en décembre 1989 à l'École polytechnique de l'Université de Montréal. Ces événements n'ont pas été qualifiés de faits divers par les journaux locaux et encore moins par les membres de la communauté universitaire de Montréal. Toutefois, à l'étranger, en dehors de ce qu'on pourrait appeler le "cercle de résonance", ces événements se retrouveront vraisemblablement dans la colonne des faits divers avec un titre accrocheur qui pourrait être: «Un tireur fou abat 16 étudiantes à Montréal!* ou *Un anti-féministe tire sur des étudiantes!* Inversement, un drame similaire, s'il se produit ailleurs, se retrouvera dans les dernières colonnes des faits divers d'ici 9 .

7 Michel Maffesoli, ane forme d'agrégation tribale*, Fait divers, Autrement, $\mathrm{n}^{\circ} 98$, avril 1988, p. 91.

8 Georges Auclair, Le mana quotidien: structures et fonctions de la cbronique des faits divers, Paris, Anthropos, coll. "Sociologie et connaissance *, 1970, p. 183.

9 le journal de Montréal du jeudi 15 novembre 1990 en page 5 rapporte un événement du même type que le drame survenu à l'École polytechnique 
14

Le fait divers est incontestablement le type d'informations privilégiẻ par les masses et cela pour plusieurs raisons: écrit dans un langage simple, familier, souvent illustré, il est à la portée de tous, il demeure près des préoccupations familières du lecteur. Une autre caractéristique du fait divers est qu'il raconte les drames des gens ordinaires. Gens ordinaires dont on parle bien peu en dehors de ces récits de transgression. En voulant retrouver les traces de l'opinion publique au XVII' siècle, l'historienne Arlette Farges remarque: «Le peuple décrit l'est en général dans sa manifestation corporelle: on parle de lui lorsqu'il acclame et crie: "Vive le Roi!", ou dès qu'il se bat au coin des rues c'est-à-dire entre violence, rixe, transgression et émotion." 10 Il en est de même pour les faits divers, ils racontent les drames vécus puisés à même la vie privée des gens ordinaires et le lecteur peut se reconnaitre dans chacune de ces histoires qui, au fond, pourrait être la sienne.

Cependant, les journaux qui tablent sur le fait divers pour accrocher l'œil du consommateur ou qui en font une spécialité sont souvent taxés de sensationnalisme, de voyeurisme, de presse à scandale, etc. Pourtant, si on arrive à surmonter l'effet de scandale, les faits divers peuvent s'avérer être pour l'ethnologue, le sociologue ou l'historien de formidables révélateurs des tensions sociales (conflit interethnique), des normes et des interdits (inceste, homosexualité, délit sexuel des prêtres), des peurs (peur du sida, peur des agressions chez les personnes âgées) et des superstitions de toutes sortes. Michel Maffesoli, dans sa contribution au numéro de la revue Autrement consacré aux faits divers mentionne que le fait divers "dit à la fois le conformisme et la dérision de ce conformisme; il rend bien compte de la duplicité (double, duple) qui structure toute existence sociale " ${ }^{11}$.

Alimenté à même l'aléatoire de la vie quotidienne des gens ordinaires, le fait divers dresse le portrait-robot de l'autre, de cet

l'an dernier: *En Nouvelle-Zélande, un dément tue 11 personnes. " Cette nouvelle, bien qu'attirante par son côté morbide et spectaculaire, n'a pas la même résonance pour le lecteur québécois que le drame survenu à l'École polytechnique de Montréal et cela du seul fait qu'elle vient d'ailleurs. Il en est de même pour le fait divers américain proposé pour ce numéro; bien qu'horrible, il ne frappe pas de la même manière que ce drame publié en première page le 11 mai 1992 dans Le journal de Québec: "Longueuil, un ado exécute sa famille. .

10 Arlette Farges, Dire et mal dire, Paris, Seuil, 1992, p. 32.

11 Michel Maffesoli, op. cit., p. 93. 
autre qui transgresse les lois. Il ne faut pas perdre de vue que la mise en récit d'un événement n'est jamais figée et définitive. Maffesoli parle du fait divers comme de .l'une des multiples formes d'agrégation sociale, un des fils qui permettent aux personnes de se rencontrer, de communier . Pour lui,

c'est son double aspect réel (véridique) et redondant dans l'image qui en est diffusée, qui en fait un vecteur privilégié de communion: le fait divers, après avoir été événement puis image, devient commentaire d'un petit groupe, occasion de parole commune ${ }^{12}$.

Tout discours, quel qu'il soit, est inscrit dans un cycle de réactualisation dont les énonciateurs sont en nombre variable et dont les formes d'expression sont multiples. En effet, la version imprimée d'un fait divers n'est jamais que l'un des états passagers d'un discours qui se réactualise dans le cadre de son cycle de réactualisation. La dynamique en est la transmission. Un événement mis en récit fera donc l'objet de multiples réactualisations (conversation, rumeurs, anecdotes, etc.), il sera désormais accessible par le récit qu'on en fera. Ces réactualisations constitueront autant d'états de récits différents.

Au départ de cette chaîne narrative, il y a un événement socio-historique racontant une transgression. Cet événement sera raconté une première fois dans un journal. Nous en aurons une première trace écrite, un premier état. Parallèlement à sa vie "écrite ", le récit aura aussi une vie *orale*. On se racontera ce qu'on a lu, vu ou entendu raconter. On se rappellera cette histoire, on la donnera en référence, on la citera en exemple. Le récit sera peu à peu enrichi de récits similaires. Chaque récit d'événements vécus s'approprie en quelque sorte la réalité. Pratique discursive conformiste, véhicule des idées reçues, récit de transgression mettant en scène des gens ordinaires sur une toile de fond véridique, le fait divers se veut une compréhension de l'aléatoire de la vie humaine, à la fois expression de la rupture du quotidien banal et mainmise passagère sur l'incontrôlable. 